\title{
Depression und Angst bei Angehörigen
}

Nahe Angehörige und Freunde sind sehr wichtig für Krebspatienten. Sie selbst sind können aber durch die Anforderungen der Betreuung psychisch belastet sein. Die Copingstrategien der Patienten und ihre Einschätzung der Prognose beeinflussen das Ausmaß von Angst und Depression bei den Bezugspersonen. Das zeigte die Auswertung von Basisdaten, die am Zentrum für Palliativversorgung des General Hospital Cancer Center in Boston, MA/USA, erhoben wurden. Aus der Gesamtheit der Patienten einer randomisierten Studie zur frühen Palliativversorgung wurden 275 Patienten für die Analyse ausgewählt, bei denen maximal acht Wochen zuvor die Primärdiagnose eines unheilbaren Lungen- oder gastrointestinalen Tumors gestellt wurde und die noch keine Therapie erhalten hatten.

Der Anteil der Personen mit Depression war bei den Bezugspersonen und $\mathrm{Pa}$ - tienten gleich groß (16,4\% bzw. $21,5 \%$, $\mathrm{p}=0,13)$, Bezugspersonen berichteten aber signifikant häufiger über Angst (42,2 vs. 28,4\%; p < 0,001). Diese hohe Rate könnte damit zusammenhängen, dass die Betreuenden Sorge haben, sie könnten den auf sie zukommenden Anforderungen nicht gewachsen sein.

Die Copingstrategien der Patienten hatten deutliche Auswirkungen auf das Befinden der Angehörigen. Setzten die Patienten bevorzugt die Copingstrategie „Akzeptanz“ ein, war die Neigung der Betreuungspersonen zu Depression geringer $(\mathrm{p}<0,001)$. Im Gegensatz dazu ging die an sich wünschenswerte Strategie „emotionale Unterstützung“ bei den Angehörigen verstärkt mit Depression einher ( $p=0,001)$, während gleichzeitig die Neigung zu Angst verringert war $(\mathrm{p}<0,001)$. Das bedeutet: Geben Angehörige und Freunde eine warme emotionale Unter-

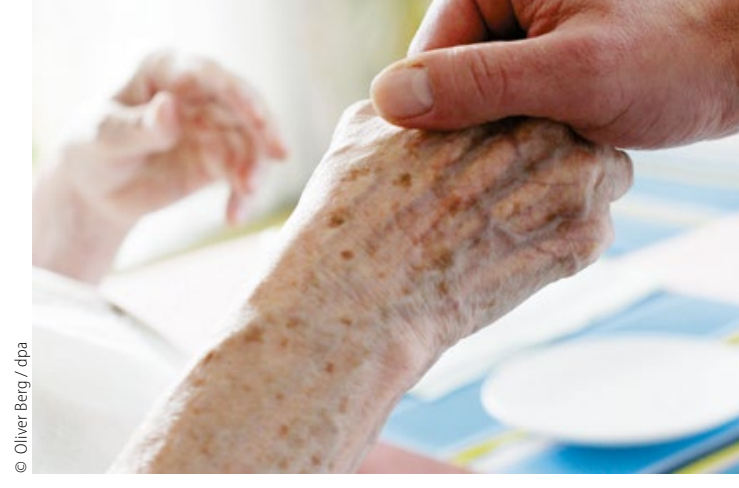

Angehörige sind eine wichtige Stütze für Patienten mit unheilbaren Erkrankungen.

stützung, kann das zwar die Angst mindern, hinterlässt aber bei den Angehörigen selbst ein Gefühl von Traurigkeit.

Die Einschätzung der Prognose durch den Patienten selbst spielte ebenfalls eine wichtige Rolle. Berichteten die Patienten, das primäre Behandlungsziel sei „den Krebs zu heilen“, führte dies bei den Bezugspersonen häufiger zu Depression $(\mathrm{p}=0,03)$.

Brigitte Schalhorn

Nipp RD et al. Factors associated with depression and anxiety symptoms in family caregivers of patients with incurable cancer. Ann Oncol. 2016; 27(8):1607-12.

\section{kurz notiert}

\section{Was die Metastasenbildung in der} Lunge beeinflusst

Ein amerikanisches Forscherteam fand heraus, dass in der Lunge gute Bedingungen für die Metastasierung vorherrschen. Es zeigte sich, dass Sauerstoff die Antitumoraktivität von T-Zellen unterdrückt. So können Tumorzellen, die in die Lunge vorgedrungen sind, dem Angriff des Immunsystems entkommen und Metastasen bilden. Verantwortlich hierfür sind Prolylhydrolase-Domäne-Proteine (PHD), sauerstoffempfindliche Proteine, die in den T-Zellen übermäßige Immunantworten auf harmlose Partikel verhindern sollen. Allerdings ermöglicht dieser Schutzmechanismus zirkulierenden Tumorzellen, in der Lunge Fuß zu fassen. PHD-Proteine fördern die Entwicklung regulatorischer T-Zellen, die die Aktivität anderer Komponenten des Immunsystems unterdrücken. Außerdem limitieren sie die Entwicklung inflammatorischer T-Zellen und hemmen deren Fähigkeit zur Produktion von Molekülen, die an der Abtötung von Krebszellen beteiligt sind. Eine pharmakologische Inhibierung von PHDProteinen könnte die Metastasenbildung in der Lunge verhindern und die Wirksamkeit einer Krebsimmuntherapie durch adoptiven Zelltransfer verbessern, so die Wissenschaftler [Clever D et al. Cell. 2016;166(5):1117-31].

Judith Neumaier

\section{Multiples Myelom \\ Lenalidomid in jedem Alter sinnvoll}

Kann bei Patienten mit multiplem Myelom (MM) keine Stammzelltransplantation erfolgen, lohnt sich eine kontinuierliche Therapie mit Lenalidomid plus niedrig dosiertem Dexamethason (Rd). Dies gilt den Resultaten einer aktuellen Studie zufolge für jedes Lebensalter. An der Studie teilgenommen hatten 1.623 Patienten mit neu diagnostiziertem MM. Von ihnen waren 567 (35\%) älter als 75 Jahre. Randomisiert erhielten die Studienteilnehmer Rd bis zum Krankheitsprogress (Rd kontinuierlich) oder Rd für 72 Wochen (18 Zyklen; Rd18) oder Melphalan, Prednison und Thalidomid (MPT; 72 Wochen).

Die kontinuierliche Rd-Therapie reduzierte im Vergleich zu MPT das Risiko für Progression oder Tod bei der Gesamtkohorte um $31 \%$ (Hazard Ratio [HR] 0,69; $\mathrm{p}<0,001)$, bei Patienten $\leq 75$ Jahren um $36 \%(\mathrm{p}<0,001)$ und bei denen, die $>75$ Jahre alt waren, um $20 \%(\mathrm{p}=0,084)$.

Auch das Gesamtüberleben (OS) war unter kontinuierlicher Rd-Therapie signifikant länger als unter MPT: Über alle Pa- tienten hinweg mehr als zehn Monate ( 58,9 vs. 48,5 Monate; HR $0,75,95 \%$-Konfidenzintervall [95\%-KI] 0,62-0,90). Bei den über 75-Jährigen gab es eine Differenz von mehr als 14 Monaten (median 52,3 vs. 37,8 Monate; HR 0,72, $95 \%$-KI 0,54-0,96), bei den höchstens 75 Jahre alten Patienten betrug sie rund fünf Monate ( 60,9 vs. 55,3 Monate; HR 0,76, 95\%-KI 0,60-0,96).

Das progressionsfreie Überleben war unter Rd18 und unter MPT vergleichbar. Unter Rd18 war das OS marginal geringer als unter kontinuierlicher Rd-Therapie.

Nebenwirkungen vom Grad 3/4 traten in beiden Altersgruppen unter kontinuierlicher Rd-Therapie vergleichbar häufig auf. Bei den älteren Patienten wurde jedoch häufiger die Lenalidomid-Dosis reduziert.

Kathrin von Kieseritzky

Hulin C et al. Updated Outcomes and Impact of Age With Lenalidomide and Low-Dose Dexamethasone or Melphalan, Prednisone, and Thalidomide in the Randomized, Phase III FIRST Trial. J Clin Oncol. 2016; Jun 20 (Epub ahead of print). 